\title{
Tratamento Endovascular do Aneurisma da Aorta Abdominal por Via Percutânea e Anestesia Local - One Day Surgery
}

\section{Percutaneous Endovascular Aortic Repair with Local Anesthesia - One Day Surgery}

\author{
Joel SOUSA ${ }^{1,2}$, Daniel BRANDÃO ${ }^{1,2}$, Paulo BARRETOํ, Joana FERREIRA ${ }^{1}$, José ALMEIDA-LOPES ${ }^{1,2}$, \\ Armando MANSILHA $\triangle 1,2$ \\ Acta Med Port 2016 Jun;29(6):381-388 - http://dx.doi.org/10.20344/amp.7715
}

\section{RESUMO}

Introdução: Avaliar os resultados do tratamento endovascular do aneurisma da aorta abdominal (EVAR) por via percutânea e anestesia local, segundo o conceito de one day surgery.

Material e Métodos: Análise retrospetiva, unicêntrica dos doentes com doença aneurismática aorto-ilíaca, consecutivamente submetidos a tratamento endovascular do aneurisma da aorta abdominal por via percutânea (pEVAR) pela técnica de Preclose, seguindo critérios de ambulatorização com pernoita após o procedimento. O sucesso técnico, exclusão do saco aneurismático, endoleak, reintervenção e tempo de internamento foram avaliados.

Resultados: Vinte doentes consecutivos (todos homens, idade média 74,65 anos) foram tratados por pEVAR e anestesia local, dos quais $95 \%$ (19) apresentavam aneurisma da aorta abdominal e 5\% (1) aneurisma da artéria ilíaca comum. Todos os implantes foram realizados com sucesso, com uma taxa de endoleak inicial de $10 \%$ (2), à custa de um endoleak 1a corrigido intraoperatoriamente com sucesso, e um endoleak 2a diagnosticado na primeira angio-tomografia computorizada pós-operatória, que selou espontaneamente no controlo aos 6 meses. O sucesso técnico inicial do encerramento percutâneo foi de 97,5\%, com um caso reportado de pseudo-aneurisma femoral, corrigido posteriormente por injeção percutânea de trombina. A mediana de internamento foi de 1 dia [1-10], com follow-up médio de 11,4 meses [1-36]. A reintervenção e mortalidade são de $0 \%$ no período descrito.

Conclusão: $O$ tratamento ambulatório do aneurisma da aorta abdominal por via endovascular com acesso percutâneo segundo o nosso modelo de one day surgery é inovador, seguro e eficaz, respeitando os critérios de seleção.

Palavras-chave: Aneurisma da Aorta Abdominal; Implante de Prótese Vascular; Procedimentos Cirúrgicos Ambulatórios; Procedimentos Endovasculares.

\section{ABSTRACT}

Introduction: To evaluate the results of the abdominal aortic aneurism endovascular treatment (EVAR), percutaneously and with local anesthesia, according to the concept of one day surgery.

Material and Methods: Unicentric, retrospective analysis of patients with aorto-iliac aneurysmal disease, consecutively treated by EVAR with percutaneous access trough the Preclose technique ( $p E V A R$ ), according to the outpatient criteria, with one overnight stay in the hospital. The technical success, exclusion of the aneurysmal sac, endoleak, re-intervention and mortality were evaluated.

Results: Twenty consecutive patients (all male; mean age 74.65 years) were treated by EVAR with percutaneous access and local anesthesia, from which $95 \%$ (19) presented with abdominal aortic aneurysm and $5 \%$ (1) common iliac aneurysm. All implants were sucessfully performed, with an initial endoleak rate of $10 \%$ (2), determined by one type 1a endoleak successfully corrected intraoperatively and one type $2 \mathrm{a}$ endoleak diagnosed in the first imaging control, which sealed spontaneously on the second control. Initial technical success for percutaneous closure was $97.5 \%$, with one case reported of femoral pseudo-aneurism, posteriorly treated by percutaneous thrombin injection. Median length of stay was one day [1-10], with a mean follow-up of 11.4 months [1-36]. Both the reintervention and mortality rate are $0 \%$ for the selected period.

Conclusion: Our one day surgery model for the outpatient treatment of abdominal aortic aneurysm by the pEVAR technique is innovative, safe and effective, as long as the selection criteria are respected.

Keywords: Ambulatory Surgical Procedures; Aortic Aneurysm, Abdominal; Blood Vessel Prosthesis Implantation; Endovascular Procedures.

\section{INTRODUÇÃO}

A primeira correção endovascular de aneurisma da aorta (EVAR) foi realizada em 1987 por Volodos et al, ${ }^{1}$ tendo a sua consagração enquanto opção terapêutica para o aneurisma da aorta abdominal surgido mais tarde, em 1991, com a publicação de Parodi et al. ${ }^{2}$ Desde então que sua aceitação pela comunidade clínica tem vindo a crescer progressivamente, com diversos estudos revelando menores taxas de internamento e morbilidade e mortalidade pós- operatórias, de tal forma que nos últimos 10 anos praticamente se tornou o gold-standard no tratamento desta patologia. ${ }^{3,4}$

Devido ao grande diâmetro das bainhas utilizadas neste tipo de implantes, o procedimento requeria inicialmente exposição uni ou bilateral da artéria femoral comum, de modo a permitir o correto controlo e manipulação da mesma durante a implantaçao do material (oEVAR). ${ }^{5}$ Embora este

\footnotetext{
1. Serviço de Angiologia e Cirurgia Vascular. Hospital CUF Porto. Porto. Portugal.

2. Faculdade de Medicina da Universidade do Porto. Porto. Portugal.

$\bowtie$ Autor correspondente: Armando Mansilha. vascular.mansilha@gmail.com

Recebido: 04 de abril de 2016 - Aceite: 21 de abril de 2016 | Copyright $\odot$ Ordem dos Médicos 2016
} 
tipo de exposição seja considerada uma intervenção minor no âmbito da Cirurgia Vascular, esta não é desprovida de complicações e riscos. De facto, complicações associadas à exposição cirúrgica da artéria femoral estão descritas em $14-22 \%$ dos casos, as quais variam em gravidade, passando do simples hematoma inguinal ou linfocelo, até trombose, dissecção arterial, lesão do nervo femoral, infeção ou mesmo necrose da ferida cirúrgica. ${ }^{6,7}$ Inevitavelmente, todos estes resultam numa diminuição da mobilidade no pós-operatório, cicatrização inadequada da ferida cirúrgica e consequente internamento mais prolongado, o que levou à procura de alternativas mais eficazes.

Os dispositivos de encerramento percutâneo dependentes de suturas foram desenvolvidos inicialmente para permitir hemóstase rápida e segura da artéria de acesso após procedimentos que requeressem o uso de bainhas de diâmetros baixos (compreendidos entre 5 e 10 F). ${ }^{8}$ A sua eficácia neste tipo de aplicações foi notável, pelo que foram progressivamente aplicados no encerramento de orifícios de arteriotomia de maiores dimensões. Em 1999, Haas et al descreveu, pela primeira vez, o EVAR por via percutânea (pEVAR), demonstrando a possibilidade de encerramento de grandes orifícios de acesso utilizando o dispositivo de encerramento percutâneo por sutura Prostar XL (Abbott Vascular, Redwood City, CA), segundo uma técnica que viria a tornar-se conhecida como a técnica de Preclose. ${ }^{9}$ Mais tarde, em 2007, esta técnica foi igualmente descrita para o dispositivo de encerramento percutâneo de segunda geração Proglide (Abbott Vascular, Redwood City, CA). ${ }^{10}$

A aplicação destes dispositivos de forma eficaz permitiu assim a realização de implantes sem qualquer exposição arterial cirúrgica, com todas as vantagens que a isto acrescem, nomeadamente menor morbilidade pos-operatória, menores complicações locais e naturalmente, menor tempo de internamento. ${ }^{11-13}$

Numa tentativa de avaliar a real utilidade do pEVAR, vários estudos unicêntricos e ensaios não randomizados foram publicados, observando-se em todos outcomes favoráveis e comparáveis ao oEVAR, com possibilidade de controlo hemostático adequado do orifício de punção. A isto acrescem algumas vantagens observadas neste subgrupo, com menores tempos de internamento, menores perdas hemáticas e menores complicações relacionadas com o procedimento, o que naturalmente se traduziu num aumento global da satisfação dos doentes, quando em comparação com a opção aberta. . $^{11,13-15}$

Em 2013 surgiu o primeiro ensaio prospetivo, multicêntrico, randomizado e controlado, cujo propósito era delinear os riscos e benefícios do pEVAR quando em comparação com a exposição femoral clássica - o PEVAR trial. ${ }^{8}$ Este ensaio demonstrou uma taxa de sucesso técnico de $94 \%$ nos encerramentos com Proglide e $88 \%$ daqueles com ProStar XL, para $98 \%$ verificado na exposição femoral. ${ }^{8}$ A subanálise da taxa de falência revelou não inferioridade dos encerramentos com proglide ( $6 \%$ falência), versus exposição femoral aberta (10\% falência), facto que não se verificou com o Prostar XL, cuja aplicação esteve asso- ciada a taxas de falência superiores à abordagem femoral (12\% falência). ${ }^{8,12}$ Ambos os sistemas de encerramento percutâneo permitiram reduções significativas dos tempos de hemóstase e duração do procedimento, com tendências favoráveis (embora sem significância estatística) no que diz respeito à perda hemática, dor inguinal e qualidade de vida geral, quando em comparação com a exposição femoral clássica. $^{8}$

Embora a literatura descreva uma diminuição do tempo de internamento com o recurso à técnica de encerramento percutâneo, a magnitude desta redução quando em comparação com o oEVAR não era tão alta quanto inicialmente expectável, com diminuições do tempo médio de internamento de 3,5 para 2,6-2,7 dias nas séries em que isto foi avaliado. ${ }^{15,16}$

Numa tentativa de otimizar este período de internamento, e uma vez que a grande maioria das complicações do acesso percutâneo têm sido documentadas intraoperatoriamente ou nas primeiras após o procedimento, estudos para avaliar a eficácia e segurança do pEVAR em ambulatório têm vindo a ser conduzidos. ${ }^{4,16,17}$

Um estudo por Dosluoglu et $a^{16}$ visou avaliar a possibilidade de ambulatorização dos doentes com aneurisma da aorta abdominal (AAA) tratados por EVAR. A sua série demonstrou que aproximadamente $33 \%$ dos doentes tratados por pEVAR podem ter alta para o domicílio com segurança depois de um período de observação de 6 horas, desde que o procedimento tenha decorrido sem complicações, apresentem boa capacidade funcional e não sejam portadores de comorbilidades de risco. Para esta baixa taxa de ambulatorização contribuiu largamente a técnica anestésica escolhida, uma vez que a grande maioria dos doentes $(81 \%)$ foi intervencionada sob anestesia geral, limitando este processo. ${ }^{16}$

Com base nestas informações, e tendo em conta a possibilidade real de ocorrência de complicações arteriais tardias, assim como a própria preferência do doente, instituiuse no nosso departamento um protocolo ambulatorial de pEVAR pela técnica de Preclose, sob anestesia local, com uma pernoita após intervenção, num modelo de one day surgery.

Este trabalho pretende avaliar os resultados da nossa experiência.

\section{MATERIAL E MÉTODOS Desenho e metodologia}

Procedeu-se a uma análise retrospetiva e unicêntrica dos doentes com doença aneurismática aorto-ilíaca, consecutivamente submetidos a EVAR com acesso percutâneo e anestesia local segundo a técnica de Preclose, desde que se iniciou a prática desta técnica na nossa instituição.

Todos os casos foram eletivos, tendo casos emergentes ou ruturas sido excluídos desta série.

Foram avaliadas as características demográficas dos doentes, assim como a sua apresentação clinica, o diâmetro do saco aneurismático, intercorrências intraoperatórias e sucesso técnico do encerramento percutâneo. 
A exclusão do saco aneurismático, a taxa de endoleak, a necessidade de reintervenção e o tempo de internamento foram também avaliados.

\section{Procedimento}

O planeamento cirúrgico dos casos e a seleção da endoprótese foi realizada por Cirurgiões Vasculares experientes com base nas características anatómicas do doente, tendo em todos os casos sido respeitadas as IFU's (instructions for use) do material utilizado.

Em todos os doentes foi planeado um acesso percutâneo ecoguiado com encerramento pela técnica de Preclose / double proglide, desde que reúnidas as condições anatómicas adequadas para tal. A presença de aneurisma da artéria femoral comum ou doença aterosclerótica grave com oclusão total foram considerados critérios de exclusão. A presença de calcificação circunferencial da artéria de acesso não foi contraindicação à utilização do acesso percutâneo, desde que o estudo ecográfico pré-operatório revelasse a presença de uma zona adequada à punção. O diâmetro arterial do vaso de acesso foi também avaliado em todos os procedimentos, de modo a garantir que o acesso e encerramento percutâneos fossem aplicados apenas em doentes com condições anatómicas para tal. $\mathrm{O}$ excesso de peso não foi considerado critério de exclusão.

Todos os doentes foram intervencionados sob anestesia local com lidocaína a $2 \%$ e ligeira sedação consciente. Cooperação ativa do doente foi requerida durante as intervenções, com solicitação de apneia durante as angiografias intraoperatórias.

\section{Conceito de one day surgery}

Segundo o nosso modelo de one day surgery, todos os doentes permanecem internados durante uma noite após a intervenção, sob vigilância em unidade de cuidados intermédios, sendo reavaliados pela equipa cirúrgica responsável até às 24 horas após. Todos os doentes com resultados angiográficos intraoperatórios satisfatórios e que à reavaliação se apresentem clinicamente bem, com boa função renal sem indicação para hidratação intravenosa, sem comorbilidades médicas major e sem complicações locais do encerramento percutâneo, têm alta para o domicílio, após the serem fornecidos contactos de apoio e explicados sinais de alarme perante os quais devem ser reavaliados.

A primeira reavaliação ocorre, em média, às duas semanas após a intervenção, com realização de angio tomografia computorizada (angioTC) abdomino-pélvico ao primeiro e sexto meses pós-operatórios. Perante ausência de endoleak ou expansão de saco aneurismático aos seis meses, são reavaliados a cada 12 meses por angioTC ou ecoDoppler abdominal, segundo o critério do cirurgião responsável.

\section{Análise estatística}

A análise dos dados foi realizada utilizando o software SPSS 22.0 (SPSS Inc, Chicago, III). Todos os valores de $P$ foram considerados estatisticamente significativos desde que $<0,05$.

\section{RESULTADOS}

Um total de 20 doentes consecutivos foram referenciados e tratados na nossa instituição. Todos os doentes intervencionados eram do sexo masculino, com uma idade média de 74,65 anos [61-88]. Nesta amostra, 55\% (11) dos doentes eram fumadores, $75 \%$ (15) sofriam de HTA, 15\% (3) de DM tipo II, 60\% (12) de dislipidemia e 30\% (6) de doença arterial coronária (Tabela 1). Nenhum dos doentes apresentava doença renal crónica (TFG $<90 \mathrm{~mL} / \mathrm{min} / 1.73$ $\mathrm{m}^{2}$ ).

Dos 20 doentes tratados, 95\% (19) apresentavam aneurisma da aorta abdominal e 5\% (1) aneurisma da artéria ilíaca comum, tendo todos sido tratados por EVAR com acesso por via percutânea (Fig.s 1, 2 e 3). Dos aneurismas da aorta abdominal, $11 \%$ (2) eram saculares, apresentando os restantes (17) uma conformação fusiforme. Aneurismas concomitantes de outros territórios vasculares foram encontrados em $20 \%$ dos doentes (4) - dois casos de aneurisma poplíteo, um caso de aneurisma da aorta torácica e um caso de aneurisma da artéria femoral superficial, sem atingimento da artéria femoral comum.

Calcificação femoral circunferencial foi encontrada em $9,5 \%$ (2) dos doentes. Contudo, o acesso ecoguiado permitiu a identificação de uma zona adequada à punção e aplicação do dispositivo de encerramento percutâneo em todos os casos.

Todos os doentes foram intervencionados sob anestesia local com lidocaína a $2 \%$ na zona de punção em associação com sedação consciente.

Todos os implantes endovasculares foram realizados com sucesso, com uma taxa de endoleak inicial de $10 \%$ (2), à custa de um caso de endoleak tipo 1a corrigido com sucesso intraoperatoriamente pelo recurso ao implante de um cuff aórtico, e um caso de endoleak tipo 2a diagnosticado no primeiro controlo imagiológico pós-operatório, o qual selou espontaneamente no angio-TC de controlo aos seis meses. Em nenhuma das situações houve necessidade de conversão para correção aberta.

Constatou-se estabilidade clínica às 24 horas após o procedimento em todos os doentes intervencionados, sem qualquer morbilidade cardíaca, lesão renal aguda ou isquemia de membro a relatar.

O sucesso técnico inicial do encerramento percutâneo foi alcançado em 97,5\% (39 acessos). Em um caso, constatou-se pseudo-aneurisma femoral diagnosticado ecograficamente às seis horas após o procedimento, tendo o doente sido submetido a correção percutânea do mesmo por injeção ecoguiada de trombina, com total reversão, e sem necessidade de intervenção cirúrgica (complicação Clavien-Dindo tipo I).

Não foi encontrada relação estatisticamente significativa entre a presença de calcificação circunferencial da artéria de acesso, e as complicações descritas ( $p$ $>0,05)$. Em nenhum caso se constatou estenose arterial pós-encerramento, nem qualquer lesão iatrogénica 


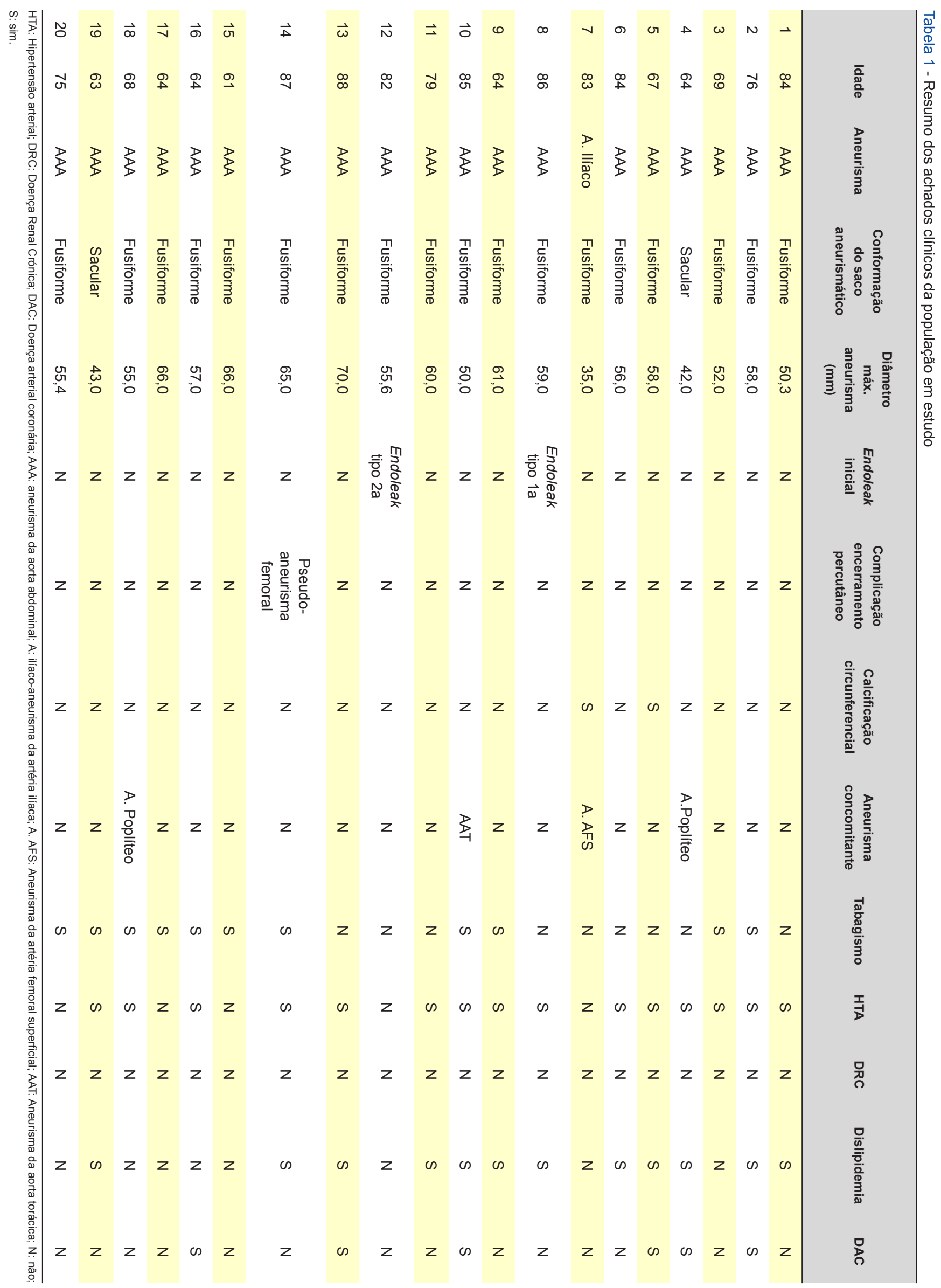




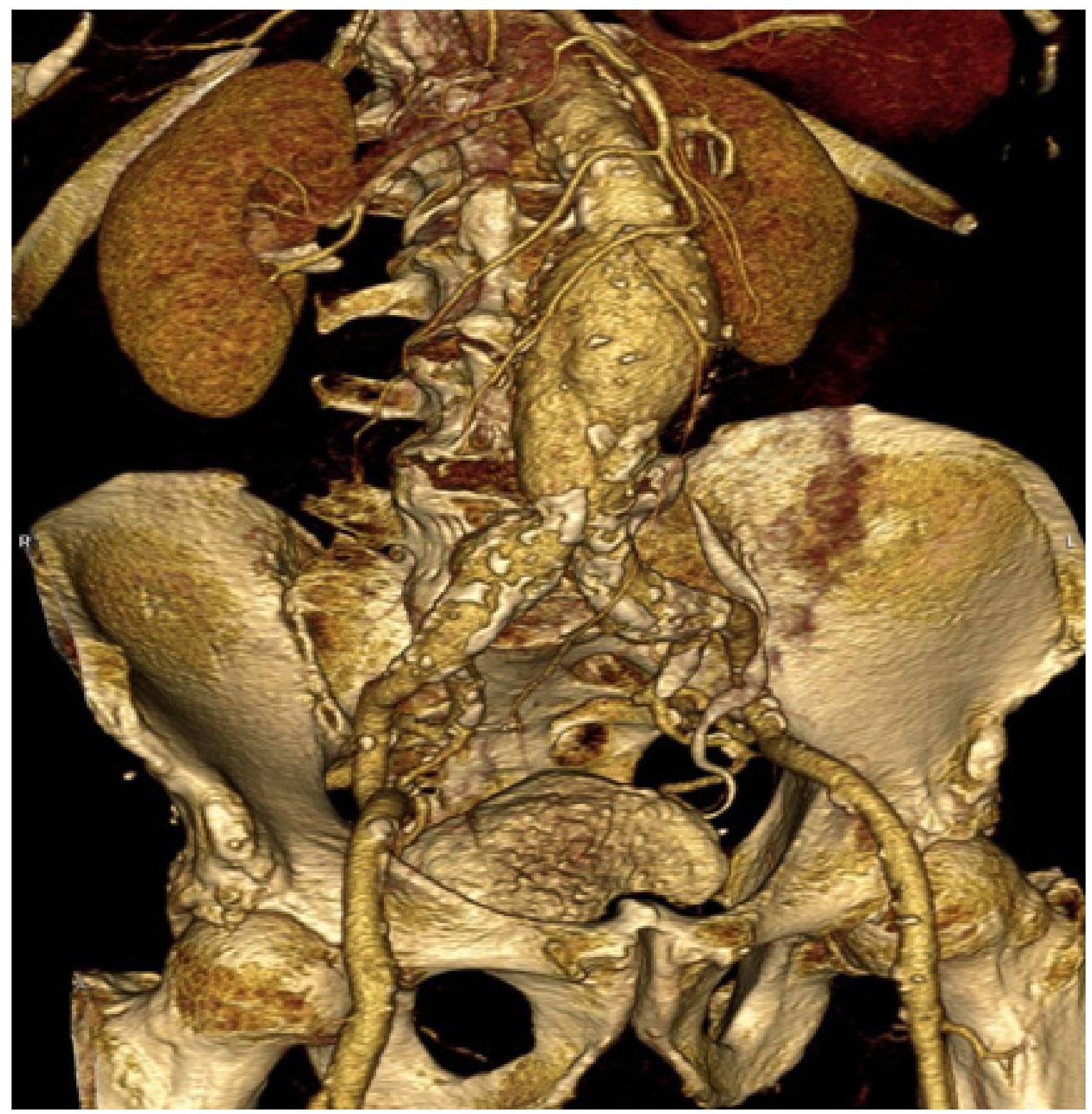

Figura 1 - Reconstrução tridimensional em angioTC de aneurisma da aorta abdominal

hemodinamicamente significativa que requeresse intervenção.

A mediana de internamento foi de um dia [1-10 dias], com alta para o domicílio às 24 horas em $95 \%$ dos doentes. Em um caso, pela complicação descrita anteriormente, não foi possível alta neste regime ambulatorial, tendo o doente permanececido internado um total de 10 dias para correção percutânea do pseudo-aneurisma e estabilização clínica.

Todos os doentes foram reavaliados às duas semanas, primeiro mês e sexto mês pós-operatório. Nenhuma complicação local foi documentada aquando da primeira avaliação. Nenhum caso de migração de prótese ou en- doleak de novo foi documentado aquando das avaliações imagiológicas ao primeiro e sexto meses pós-operatórios. Em um caso de endoleak tipo 2 a diagnosticado intraoperatoriamente, constatou-se selagem espontânea no angioTC de controlo ao sexto mês pós-operatório, achado que se manteve no controlo imagiológico subsequente.

O período de follow-up médio foi de cerca de 11,4 meses [1-36 meses], sem perdas para o seguimento até ao momento. Nenhum doente foi readmitido para o período de follow-up descrito. A taxa de reintervenção é de $0 \%$, não existindo mortalidade descrita, de qualquer causa, até ao momento. 


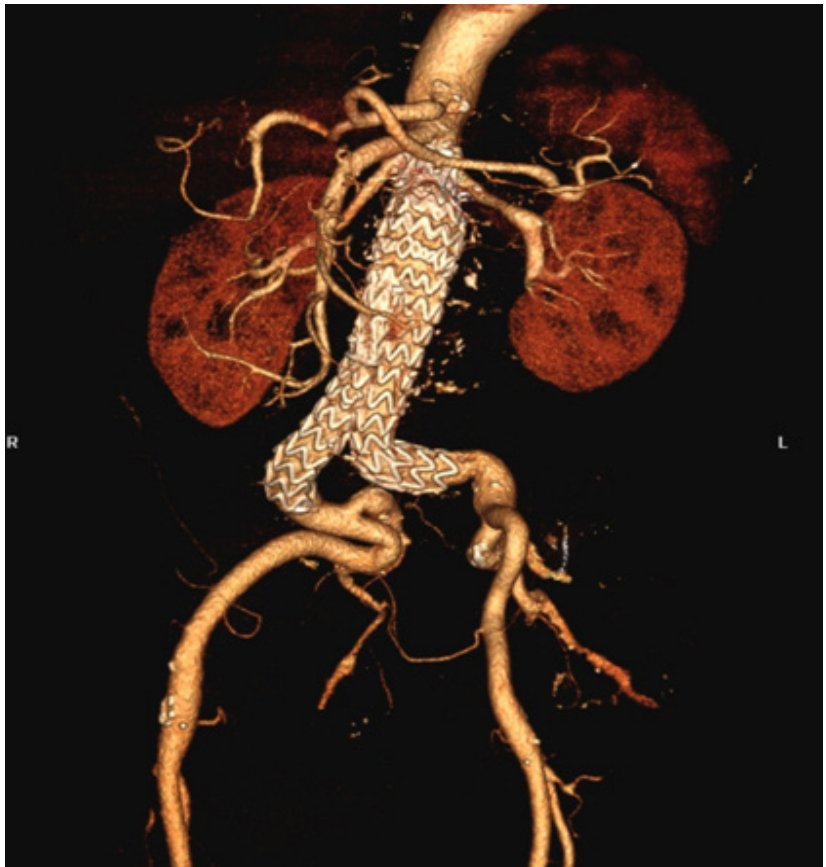

Figura 2 - Reconstrução tridimensional em angioTC pós-EVAR

\section{DISCUSSÃO}

Embora exista alguma evidência de que a redução do período de internamento se traduza em vantagens para o doente, assim como vantagens económicas para o próprio sistema de saúde, existem apenas alguns índices que demonstram que o tratamento ambulatório do aneurisma da aorta abdominal é seguro e reproductível. ${ }^{17,18}$

$\mathrm{Na}$ nossa série de casos, 20 doentes consecutivos foram tratados por EVAR, por via percutânea e sob anestesia local, com pernoita em unidade de cuidados intermédios na noite do procedimento (segundo um protocolo estandardizado), sem mortalidade, sem reintervenções, e com uma taxa de complicações mínima para o período de follow-up descrito.

Ao contrário de outras séries de pEVAR em ambulatório com protocolos estandardizados de alta no próprio dia da intervenção, ${ }^{17}$ o nosso modelo baseia-se na pernoita por uma noite na instituição. Isto porque, embora a maioria das complicações relacionadas com o encerramento percutâneo se manifestem nas primeiras horas após o procedimento, ${ }^{4}$ são raras mas possíveis as situações de complicação local tardia, que não só podem ser fonte de complicação major se não detetadas atempadamente, como também constituem motivo de readmissão, com toda a morbilidade que isto acarreta. Mais do que isso, embora nenhum dos doentes na nossa série apresentasse doença renal crónica diagnosticada pré-intervenção, é facto que a lesão renal aguda induzida por contraste apenas se manifesta às 2472 horas, facto que poderia passar despercebido perante alta em regime ambulatório imediato. ${ }^{19}$

Por essa razão, o nosso protocolo baseia-se na pernoita na instituição na noite do procedimento, garantindo assim uma maior vigilância dos parâmetros clínicos e póscirúrgicos, sem perda das características de ambulatoriza-

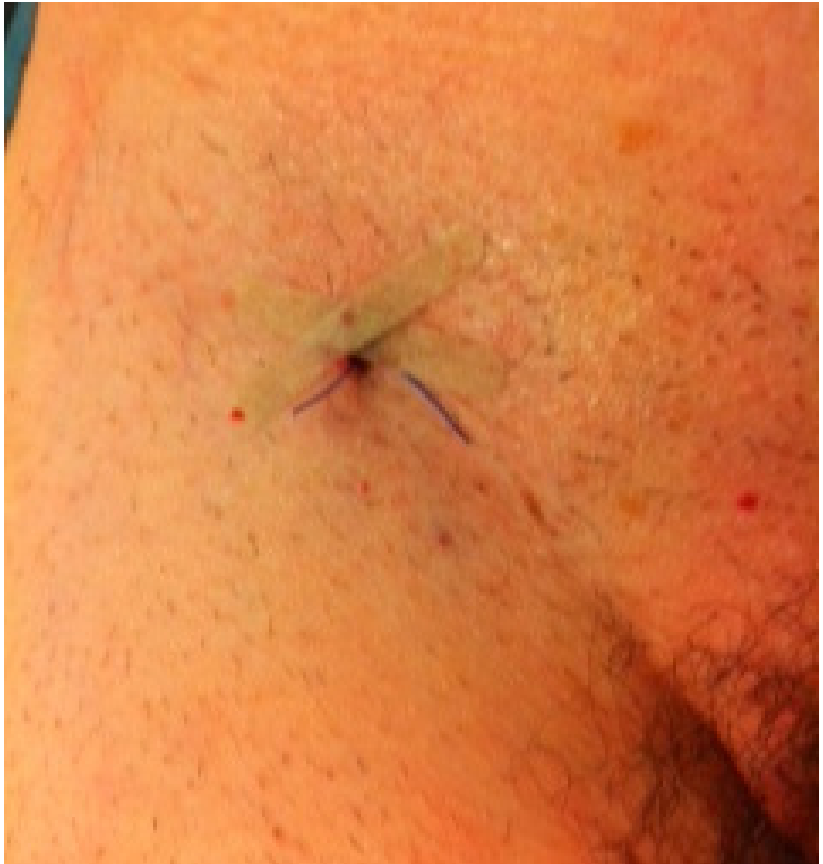

Figura 3 - Penso em zona de acesso, pós-intervenção

ção cujos benefícios clínicos têm vindo a ser demonstrados.

Também a técnica anestésica selecionada contribuiu para os resultados alcançados, à semelhança do descrito na literatura. Numa revisão de 10 estudos comparando a anestesia local à regional, envolvendo 13.459 doentes, o subgrupo submetido a anestesia local apresentou menores tempos cirúrgicos, menores períodos de internamento e menos complicações pós-operatórias. ${ }^{20}$ Embora a anestesia local possa estar associada a um desconforto durante a manipulaçao do material ou isquemia relativa de membro, estas limitações foram compensadas pela aplicação de sedação consciente pela nossa equipa anestésica, garantindo maior conforto ao doente durante todo o procedimento. Em última análise, o recurso a este modelo reduziu drasticamente as complicações relacionadas com a entubação ou curarização dos doentes, facilitando a sua alta mais precoce.

Por fim, a taxa de complicações do encerramento documentada na nossa série (2,5\% - uma complicação), é sobreponível àquela descrita em algumas das maiores revisões sistemáticas, as quais apresentam taxas tão baixas quanto $3,6 \% .^{21}$

A obtenção destes resultados está amplamente dependente da experiência do operador, ${ }^{22}$ assim como da correta seleção dos doentes.

Vários são os determinantes de sucesso ou insucesso técnico no pEVAR descritos na literatura, com maior ou menor concordância entre os diferentes estudos, e que devem ser sempre respeitados para um melhor outcome possível. Particular destaque deve ser dado à punção ecoguiada e à avaliação pré-operatória do diâmetro da artéria de acesso.

No que diz respeito à punção ecoguiada, o seu papel 
nos procedimentos percutâneos tem vindo a ser reforçado na literatura. Idealmente, o acesso à artéria femoral comum deve ser sempre conseguido pela sua parede anterior (às $12 \mathrm{~h}$ ), aproximadamente $1 \mathrm{~cm}$ proximalmente à bifurcação femoral e numa zona sem placa aterosclerótica. Não respeitar estas condições aumenta em muito o risco de complicações. Isto porque se por um lado punções demasiado altas podem estar associadas a incorporação do ligamento inguinal na sutura, com consequente risco de rutura da mesma e hemorragia potencialmente fatal aquando do início da deambulação, por outro lado, punções demasiado baixas na artéria femoral superficial, de menor diâmetro, podem ser causa de lesão da parede do vaso, com oclusão e isquemia. ${ }^{14}$ Por esta razão, o acesso ecoguiado da artéria femoral comum para a realização de pEVAR é agora virtualmente mandatório em todos os casos. ${ }^{23}$

Por outro lado, também o diâmetro do vaso de acesso parece ser um determinante major do sucesso técnico da aplicação de dispositivos de encerramento percutâneo. Isto porque, perante baixos diâmetros, o dispositivo poderá não funcionar adequadamente, uma vez que existe risco de sutura da parede posterior da artéria, levando invariavelmente a complicações. ${ }^{14}$ Por esta razão, considera-se atualmente que a aplicação destes dispositivos em artérias com $<5$ $\mathrm{mm}$ constitui um preditor de falência técnica, ${ }^{24}$ algo que foi considerado na avaliação pré-operatória dos doentes intervencionados. De realçar ainda que as mulheres, enquanto subgrupo de género, experienciam uma maior taxa de falência técnica quando em comparação com os homens, o que naturalmente se deve ao baixo diâmetro das artérias. $A$ aplicação destes dispositivos em doentes do sexo feminino adequadamente selecionados apresenta resultados sobreponíveis àqueles descritos nos grandes ensaios. ${ }^{25}$

No que diz respeito ao impacto da obesidade e calcificação da parede arterial no sucesso técnico do acesso percutâneo, ao contrário do que acontece para os determinantes previamente discutivos, a evidência é discordante. ${ }^{14}$ Por essa razão, nenhum destes fatores foi considerado critério de exclusão ao pEVAR na nossa série, e limitações inerentes à sua presença foram colmatadas pelo acesso ecoguiado, seja pela identificaçao de uma zona não calcificada em situação de calcificação arterial, seja pela possibilidade de melhor identificação dos limites do vaso de acesso na presença de maior panícula adiposa.

\section{CONCLUSÃO}

Desde que aplicada por cirurgiões treinados em doentes adequados, o recurso à técnica de EVAR com acesso percutâneo pela técnica de Preclose é segura, eficaz e está associada a uma baixa taxa de complicações locais.

O recurso à anestesia local com sedação consciente, a punção ecoguiada e a elevada experiência do grupo cirúrgico permitiu o tratamento em ambulatório do aneurisma da aorta abdominal por pEVAR, com uma elevadíssima taxa de sucesso técnico e morbilidade mínima associadas.

A decisão pela pernoita pós-intervenção, segundo um protocolo estandardizado pré-definido, permitiu identificar complicações de aparecimento mais tardio, assegurando a sua correção no mesmo período de internamento, e evitando readmissões com aumento potencial da morbilidade.

Conclui-se assim que o tratamento em ambulatório do aneurisma da aorta abdominal por pEVAR segundo o nosso modelo de one day surgery é inovador, seguro e eficaz, permitindo combinar as vantagens consagradas do tratamento ambulatorial, com uma maior vigilância pós-operatória imediata, essencial a um pós-operatório sem intercorrências.

\section{CONFIDENCIALIDADE DOS DADOS}

Os autores declaram ter seguido os protocolos do seu centro de trabalho acerca da publicação dos dados de doentes.

\section{PROTECÇÃO DE PESSOAS E ANIMAIS}

Os autores declaram que os procedimentos seguidos estão de acordo com os regulamentos estabelecidos pelos responsáveis da Comissão de Investigação Clínica e Ética e de acordo com a Declaração de Helsínquia da Associação Médica Mundial.

\section{CONFLITO DE INTERESSES}

Os autores declaram não possuir conflitos de interesse.

\section{FONTES DE FINANCIAMENTO}

O presente trabalho não foi suportado por nenhum subsídio ou bolsa.

\section{REFERÊNCIAS}

1. Volodos NL, Karpovich IP, Troyan VI, Kalashnikova YuV, Shekhanin VE Ternyuk NE, et al. Clinical experience of the use of self-fixing synthetic prostheses for remote endoprosthetics of the thoracic and the abdominal aorta and iliac arteries through the femoral artery and as intraoperative endprosthesis for aorta reconstruction. Vasa Suppl. 1991; 33:93-5.

2. Parodi JC, Palmaz JC, Barone HD. Transfemoral intraluminal graft implantation for abdominal aortic aneurysms. Ann Vasc Surg. 1991;5:491-9.

3. Dua A, Kuy S, Lee CJ, Upchurch GR, Jr., Desai SS. Epidemiology of aortic aneurysm repair in the United States from 2000 to 2010. J Vasc Surg. 2014;59:1512-7.

4. Moscato VP, O’Brien-Irr MS, Dryjski ML, Dosluoglu HH, Cherr GS, Harris LM. Potential clinical feasibility and financial impact of same-day discharge in patients undergoing endovascular aortic repair for elective infrarenal aortic aneurysm. J Vasc Surg. 2015;62:855-61.

5. Morasch MD. Percutaneous techniques for aneurysm repair. J Vasc Surg. 2006;43 Suppl A:69A-72A.

6. Lonn L, Larzon T, Van Den Berg JC. From puncture to closure of the common femoral artery in endovascular aortic repair. J Cardiovasc Surg. 2010;51:791-8.

7. Maleux G, Koolen M, Heye S. Complications after endovascular aneurysm repair. Semin Intervent Radiol. 2009;26:3-9.

8. Nelson PR, Kracjer Z, Kansal N, Rao V, Bianchi C, Hashemi H, et al. A multicenter, randomized, controlled trial of totally percutaneous access versus open femoral exposure for endovascular aortic aneurysm repair (the PEVAR trial). J Vasc Surg. 2014;59:1181-93.

9. Haas PC, Krajcer Z, Diethrich EB. Closure of large percutaneous access sites using the Prostar XL Percutaneous Vascular Surgery device. J 
Endovasc Surg. 1999;6:168-70.

10. Dosluoglu HH, Cherr GS, Harris LM, Dryjski ML. Total percutaneous endovascular repair of abdominal aortic aneurysms using Perclose ProGlide closure devices. J Endovasc Ther. 2007;14:184-8.

11. Kauvar DS, Martin ED, Givens MD. Thirty-Day Outcomes after Elective Percutaneous or Open Endovascular Repair of Abdominal Aortic Aneurysms. Ann Vasc Surg. 2016;31:46-51.

12. Malkawi AH, Hinchliffe RJ, Holt PJ, Loftus IM, Thompson MM. Percutaneous access for endovascular aneurysm repair: a systematic review. Eur J Vasc Endovasc Surg. 2010;39:676-82.

13. Kontopodis N, Tsetis D, Kehagias E, Daskalakis N, Galanakis N, Ioannou CV. Totally Percutaneous Endovascular Aneurysm Repair Using the Preclosing Technique: Towards the Least Invasive Therapeutic Alternative. Surg Laparosc Endosc Percutan Tech. 2015;25:354-7.

14. Patel PJ, Kelly Q, Hieb RA, Lee CJ. Current Status of Percutaneous Endografting. Semin Intervent Radiol. 2015;32:278-88.

15. van Dorp M, Ruyssers M, Amajoud Z, Lauwers P, Van Schil PE, Hendriks JM. Preclose Percutaneous Endurant Endografting with the Proglide Device: a Safe and Feasible Combination. Acta Chir Belg. 2015;115:219-23.

16. Dosluoglu HH, Lall P, Blochle R, Harris LM, Dryjski ML. Ambulatory percutaneous endovascular abdominal aortic aneurysm repair. J Vasc Surg. 2014; 59:58-64.

17. Lachat ML, Pecoraro F, Mayer D, Guillet C, Glenck M, Rancic Z, et al. Outpatient endovascular aortic aneurysm repair: experience in 100 consecutive patients. Ann Surg. 2013;258:754-8; discussion 8-9.

18. Al-Zuhir N, Wong J, Nammuni I, Curran G, Tang T, Varty K. Selection, thirty day outcome and costs for short stay endovascular aortic aneurysm repair (SEVAR). Eur J Vasc Endovasc Surg. 2012;43:662-5.

19. Gupta RK, Bang TJ. Prevention of Contrast-Induced Nephropathy (CIN) in Interventional Radiology Practice. Semin Intervent Radiol. 2010:27:348-59.

20. Karthikesalingam A, Thrumurthy SG, Young EL, Hinchliffe RJ, Holt PJ, Thompson MM. Locoregional anesthesia for endovascular aneurysm repair. J Vasc Surg. 2012;56:510-9.

21. Jaffan AA, Prince EA, Hampson CO, Murphy TP. The preclose technique in percutaneous endovascular aortic repair: a systematic literature review and meta-analysis. Cardiovasc Intervent Radiol. 2013;36:56777.

22. Bechara CF, Barshes NR, Pisimisis G, Chen H, Pak T, Lin PH, et al. Predicting the learning curve and failures of total percutaneous endovascular aortic aneurysm repair. J Vasc Surg. 2013;57:72-6.

23. Mousa AY, Campbell JE, Broce M, Abu-Halimah S, Stone PA, Hass SM, et al. Predictors of percutaneous access failure requiring open femoral surgical conversion during endovascular aortic aneurysm repair. J Vasc Surg. 2013;58:1213-9.

24. Bensley RP, Hurks R, Huang Z, Pomposelli F, Hamdan A, Wyers $M$, et al. Ultrasound-guided percutaneous endovascular aneurysm repair success is predicted by access vessel diameter. J Vasc Surg. 2012:55:1554-61.

25. Al-Khatib WK, Zayed MA, Harris EJ, Dalman RL, Lee JT. Selective use of percutaneous endovascular aneurysm repair in women leads to fewer groin complications. Ann Vasc Surg. 2012;26:476-82. 